\title{
PREVALENCIA DE TRASTORNO POR DÉFICIT DE ATENCIÓN E HIPERACTIVIDAD, EN PACIENTES DE 6 A 12 AÑOS, HOSPITAL INFANTIL ROBERT REID CABRAL, DURANTE EL PERÍODO NOVIEMBRE 2016-ABRIL 2017
}

\author{
Prevalence of disorder due to deficit of attention and hyperactivity, \\ patients from 6 to 12 years old, Robert Reid Cabral Children's Hospital, \\ during the period november 2016 - april 2017
}

\author{
Zuleika Morillo, MD* y Deseado S. Guzmán**
}

Recibido: 13/3/2018 Aprobado: 15/5/2018

Cómo citar: Morillo Z, Guzmán D. Prevalencia de trastorno por déficit de atención e hiperactividad, pacientes de 6 a 12 años, Hospital Infantil Robert Reid Cabral, durante el periodo noviembre 2016- abril 2017 Ciencia y Salud [Internet]. 2018 mayo 1; [citado 2018 agosto 15]; 2(2):[aprox. 0 p.]. Disponible en: https://revistas.intec.edu.do/index.php/cisa/article/view/1185

\section{Resumen}

El propósito del estudio es conocer de forma clara y objetiva la prevalencia de los pacientes atendidos en la consulta de psiquiatría de este centro hospitalario, además de determinar los especificadores, los motivos de consulta, los factores de riesgo y la evolución de los pacientes.

El trastorno por déficit de atención/hiperactividad, constituye un problema de salud para nuestro país, por lo que resulta relevante investigar sobre su prevalencia, los factores asociados y su evolución.

En la actualidad el Trastorno por Déficit de Atención Hiperactividad (TDAH) es un problema de alta incidencia en los centros educativos y debido a sus características genera repercusiones educativas de consideración que interfieren con el proceso de adaptación y aprendizaje de los niños.

\section{Metodología}

Para determinar el grado de prevalencia se utilizaron escalas y entrevistas estructuradas y se siguieron los criterios del DSM5 para realizar las presunciones diagnósticas y establecer los subtipos en las edades comprendidas entre 6 y 12 años.

\footnotetext{
* Hospital Infantil Robert Reid Cabral, República Dominicana. paidozu@hotmail.com

** Hospital Infantil Robert Reid Cabral, República Dominicana. deseado@gmail.com
}

\section{Resultados}

La prevalenciaqueseobtuvo fue de un $7.6 \%$, predominando las edades ubicadas en el rango de 6 a 8 años, siendo más frecuente en el sexo masculino y en la presencia de factores de riesgo como tabaco, alcohol, prematuridad, bajo peso al nacer y nivel socioeconómico bajo.

Palabras clave: Trastorno por déficit de atención e hiperactividad; monoparental; epilepsia; prematuridad.

\begin{abstract}
Attention deficit / hyperactivity disorder is a health problem for our country, so it is relevant to investigate prevalence, associated factors and evolution of it.

At present, attention deficit hyperactivity disorder (ADHD) is a problem of high incidence in educational centers and, due to the characteristics of the disorder, it generates significant educational repercussions that interfere with the adaptation and learning process of children.

The purpose of the study is to know clearly and objectively the prevalence of patients treated in the psychiatric clinic of this hospital, as well as to determine the specifiers, reasons for consultation, risk factors and patient evolution.
\end{abstract}




\section{Methodology}

To determine the degree of prevalence, scales and structured interviews were used and the DSM- 5 criteria were followed to make the diagnostic assumptions and establish the subtypes between the ages of 6 to 12 years.

\section{Results}

The prevalence was $7.6 \%$, predominantly between the ages of 6-8 years, being more frequent in males, and in case of the presence of risk factors such as smoking, alcohol, prematurity, low birth weight and low socioeconomic status.

Keywords: Attention deficit and hyperactivity disorder; single parent; epilepsy; prematurity.

\section{Introducción}

El trastorno por déficit de atención/hiperactividad es un trastorno de carácter neurobiológico originado en la infancia que implica un patrón de déficit de atención, hiperactividad o impulsividad, que en muchas ocasiones está asociados a un trastorno comórbido ${ }^{1}$.

El Instituto de Trastorno por Déficit de Atención/ Hiperactividad de Norte América, después de una revisión de 102 estudios a nivel mundial concluyó que, aunque no existe un consenso mundial sobre la prevalencia en niños y adolescentes, los análisis de meta-regresión han estimado la prevalencia mundial de dicho trastorno entre el 5,29\% y el 7,1\% de niños y adolescentes. La prevalencia de trastorno por déficit de atención/hiperactividad en niños muy pequeños ( $<6$ años) o más tarde en la edad adulta (mayores de 44 años) es menos estudiada ${ }^{2}$.

La etiología del trastorno por déficit de atención/ hiperactividad indica que es altamente heredable. Sin embargo, también puede ser adquirido, mientras que algunos individuos tienen una combinación de TDAH genético y adquirido ${ }^{3}$.

Los datos convergentes de la neuroimagen, la neuropsicología, la genética y los estudios neuroquímicos señalan constantemente la participación de la red frontoestriatal como un probable contribuyente a la patofisiología del TDAH. Esta red implica la corteza prefrontal lateral, la corteza cingulada anterior dorsal y el núcleo caudado y putamen.

Los factores de riesgo para la evolución del trastorno por déficit de atención/hiperactividad son los ambientales y prenatales, los cuales tienen un papel fundamental en la patogénesis del TDAH. Los factores prenatales se asocian con el estilo de vida maternal durante el embarazo; por ejemplo, la exposición prenatal al alcohol y al tabaco, que inducen a unas consecuencias tales como muy bajo peso al nacer y complicaciones del parto que provocan anomalías estructurales cerebrales, especialmente en el cerebelo.

La gran dificultad de los estudios epidemiológicos del trastorno por déficit de atención e hiperactividad reside en los métodos utilizados para aceptar el diagnóstico de la enfermedad. En República Dominicana, no existe una cifra oficial de la prevalencia de trastorno por déficit de atención/hiperactividad en la población pediátrica.

El Hospital Infantil Dr. Robert Reid Cabral, principal centro de atención médica del país y el lugar del primer servicio de psiquiatría infantil, no dispone de estudios de investigación sobre pacientes con trastorno por déficit de atención/hiperactividad atendidos en el centro, dificultando una buena atención y un seguimiento adecuado de la enfermedad.

\section{Metodología}

Se trata de un estudio prospectivo y analítico, dentro de los informes de casos y serie de casos, donde los datos se recolectarán de forma prospectiva. Está compuesto por los pacientes que asistieron a la consulta rutinaria de psiquiatría infantil, durante el período noviembre 2016-abril 2017. Se seleccionaron los pacientes que cumplían con los criterios diagnósticos del DSM-V para trastorno por déficit de atención e hiperactividad. 


\section{Resultados}

La prevalencia obtenida resulto ser de un 7,6\%. El mayor porcentaje de los pacientes correspondió al sexo masculino, con el $80.3 \%$. La edad más frecuente encontrada osciló entre 6 y 8 años, con un $68.4 \%$, seguido del rango de 9-10, con un $18.4 \%$. En cuanto a los factores de riesgo prenatal para el desarrollo del trastorno por déficit de atención e hiperactividad, el 35.5\% de los pacientes durante el embarazo se relacionó con trauma o lesión, seguido de la prematuridad (21\%), siendo el exceso de alcohol (17\%) un elemento predominante para el desarrollo del trastorno. Hablando de los factores de riesgo familiar, el $80.3 \%$ de los pacientes tiene un nivel socioeconómico bajo y el $48.7 \%$ de los pacientes tiene una familia monoparental. El 90.8\% de los pacientes tiene un rendimiento escolar insuficiente. En torno al de presentación del trastorno por déficit de atención e hiperactividad: el $68.4 \%$ es mixto, el $18.4 \%$ tiene inatención y el $13.2 \%$ padece de hiperactividad.

\section{Discusión de Resultados}

El mayor porcentaje de los pacientes correspondió al sexo masculino $(80.3 \%)$, lo que se corresponde con la mayoría de los resultados reportados por la literatura internacional, donde la prevalencia del sexo masculino ha sido predominante, pues, según Baungarnerd y col. (2012), el sexo masculino es 5:1 con respecto al femenino.

En cuanto a la edad, la más frecuente encontrada estuvo entre 6 y 8 años, con el 68.4\%, seguido del rango de $9-10$, con un $18.4 \%$. Estos datos se relacionan con la literatura, refiriendo que el sexo masculino desarrolla trastorno por déficit de atención e hiperactividad más temprano que las féminas. Datos comparables con el estudio Millenium 2014, que refiere que la edad media promedio de la aparición del trastorno es de 7,2 años.

\begin{tabular}{lcc}
\hline \multicolumn{3}{c}{ Distribución de casos rango de edad } \\
\hline Rango edad & Frecuencia & Porcentaje \\
6-8 ańos & 52 & 68.4 \\
9-10 años & 14 & 18.4 \\
11-12 años & 10 & 13.2 \\
Total & 76 & 100.0 \\
\hline
\end{tabular}

De acuerdo al lugar de procedencia de los pacientes del estudio, el $59.2 \%$ de la población estuvo representada por Santo Domingo (Gran Santo Domingo y el Distrito Nacional), seguido por la región sur (34.2\%). Tomando en cuenta que el Hospital Infantil Robert Reid Cabral está ubicado en el Distrito Nacional, demográficamente es un punto de referencia para ambas zonas, debido a su proximidad geográfica, por lo que estos datos no son concluyentes de que la mayor población con trastorno por déficit de atención e hiperactividad pertenece a las áreas descritas, por tanto, se necesita una investigación que incluya todas las regiones del país para determinar la prevalencia del trastorno a nivel nacional.

Como se ha descrito, la etiología del trastorno por déficit de atención e hiperactividad es multifactorial, ya que intervienen factores predisponentes, genéticos, familiares y ambientales.

Con relación a los factores de riesgo prenatal para el desarrollo del trastorno por déficit de atención e hiperactividad, el 35.5\% de los pacientes durante el embarazo se relacionó con trauma o lesión en el embarazo, seguido de la prematuridad (21\%), siendo el exceso de alcohol (17\%) un elemento predominante para su desarrollo. Estos datos se relacionan con otras investigaciones citadas, como la realizada en 2004 en el Hospital Palma de Mallorca, que reveló que los factores prenatales que aumentaban el riesgo de desarrollar el trastorno por déficit de atención e hiperactividad son los traumas y la genética.

Factores de riesgo prenatal 


\begin{tabular}{lcc}
\hline & Recuento & Porcentaje \\
Herencia & 11 & 14.5 \\
$\begin{array}{l}\text { Trauma o lesión } \\
\text { embarazo }\end{array}$ & 27 & 35.5 \\
Prematuridad & 16 & 21 \\
Peso bajo al nacer & 12 & 15.7 \\
Alcohol & 13 & 17 \\
Drogas & 3 & 3.9 \\
Exposición al ta- & 2 & 2.6 \\
baco & & \\
\hline
\end{tabular}

En lo concerniente a los factores de riesgo familiar del trastorno por déficit de atención e hiperactividad, el $80.3 \%$ de los pacientes tiene un nivel socioeconómico bajo, seguido de los conflictos familiares, con un $77.6 \%$. Esta información se relaciona con la investigación realizada por el Departamento de Medicina Familiar de la Universidad de Wisconsin, que resalta que los ingresos bajos en el medio familiar y el aumento de densidad de personas son factores de riesgo para el desarrollo del trastorno por déficit de atención e hiperactividad.

\begin{tabular}{lcc}
\hline \multicolumn{3}{c}{ Factores de riesgo familiar } \\
\hline & Recuento & Porcentaje \\
Nivel económico bajo & 61 & $80.3 \%$ \\
Conflictos familiares & 59 & $77.6 \%$ \\
$\begin{array}{l}\text { Pautas educativas } \\
\begin{array}{l}\text { Antecedente psiquiátrico } \\
\text { familiar }\end{array}\end{array}$ & 35 & $46.1 \%$ \\
\hline
\end{tabular}

De acuerdo a este hallazgo, cabe suponer que presentar niveles económicos bajos, conflictos familiares y antecedentes psiquiátricos familiares son factores de riesgo para el desarrollo del trastorno por déficit de atención e hiperactividad.

En cuanto al tipo de familia, el $48.7 \%$ de los pacientes tiene una familia monoparental, seguido de la familia nuclear, con un $43.4 \%$, relacionándose esta información con la literatura que describe la predisposición, según Quiroga y cols, acerca de que el trastorno por déficit de atención e hiperactividad se puede desarrollar con un vínculo inseguro y en familias disfuncionales.

Al hablar de la escolaridad de los pacientes, el $80.3 \%$ se encuentra en el nivel escolar y el $13.2 \%$ en el nivel preescolar, tomando en cuenta que la investigación comprende las edades desde los 6 a los 12 años.

Pasando al control prenatal, el $75 \%$ de la madres presentaron seguimiento durante el embarazo y un $25 \%$ no presentó ningún seguimiento obstétrico. Existen números estudios que califican los factores prenatales como elementos de riesgo para el desarrollo del trastorno por déficit de atención e hiperactividad. Según Silva D y cols, fumar durante el embarazo, la infección urinaria materna, el parto inducido y la amenaza de parto prematuro incrementan el riesgo de trastorno por déficit de atención e hiperactividad en ambos sexos ${ }^{16}$.

De acuerdo al rendimiento escolar de los pacientes, el $90.8 \%$ es insuficiente y el $9.2 \%$ es suficiente. Existe un sinnúmero de datos estadísticos que reportan en sus conclusiones, según Enriquez E y cols, que los problemas de aprendizaje, de conducta y emocionales, que se manifiestan en el ecosistema escolar son factores que inciden en la alteración de la vida escolar del niño, evidenciándose como resultado una disminución en su rendimiento escolar ${ }^{45}$.

Con relación al subtipo del trastorno por déficit de atención e hiperactividad, el $68.4 \%$ es mixto, el $18.4 \%$ tiene inatención y el $13.2 \%$ padece hiperactividad. Relacionándose esta información con diversas literaturas, según Wolraich y cols, se concluyó que la prevalencia es de un $8,7 \%$ para Carolina del Sur y de un 10,6\% para Oklahoma, evidenciándose un predominio del subtipo mixto o combinado. 


\section{Clasificación}

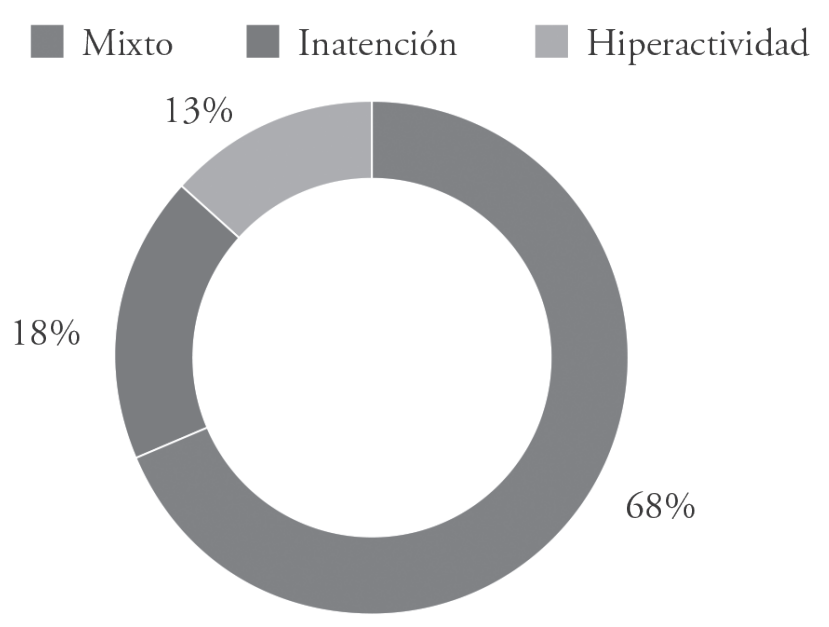

\section{Conclusiones}

La edad más frecuente determinada está entre 6 y 8 años, donde predominó el sexo masculino. El mayor índice de procedencia fue del gran Santo Domingo, seguido de la Región Sur. El factor de riesgo prenatal más relevante es un trauma o una lesión en el embarazo, seguido de la prematuridad y el alcohol. El tipo de familia más frecuente que se determinó es la familia monoparental, continuando con la familia neutral. Los factores de riesgos familiares más frecuentes son el nivel socioeconómico bajo y los conflictos familiares. La escolaridad que predominó fue el nivel escolar, seguido del nivel preescolar. En cuanto al control prenatal, el $75 \%$ de las madres fueron a chequeos y el $25 \%$ no tuvo ninguno. Con relación al rendimiento escolar, el mayor porcentaje fue insuficiente. El subtipo de trastorno por déficit de atención e hiperactividad es el mixto, seguido del tipo inatención.

\section{Recomendaciones}

Se recomienda propiciar la formación adecuada de los docentes sobre el trastorno por déficit de atención e hiperactividad, para su detección a tiempo. Además, crear una unidad trabajo con el Ministerio de Salud para el seguimiento de los niños, así como aulas calificadas con este propósito para así tener un mejor rendimiento académico. Entre las demás recomendaciones están: realizar campañas de concientización a la población general sobre los diferentes factores de riesgo, tanto profesores, padres y tutores; establecer equipos de trabajo con los diferentes equipos médicos desde el embarazo hasta después del nacimiento; propiciar las condiciones para un control prenatal adecuado; realizar investigaciones en el país que permitan establecer la prevalencia real del trastorno por déficit de atención e hiperactividad; asegurarse de contratar los profesionales y técnicos adecuados para el seguimiento y diagnóstico oportuno; garantizar la cobertura terapéutica a todos los pacientes en los hospitales.

\section{Bibliografía}

1. ADHD Institute, EEUU [internet]. Atlanta, EEUU: ADHD Institute [citado 24 marzo 2017]. Disponible en:

http://www.adhd-institute.com/ burden-of-adhd/epidemiology

2. Kytja KS, Voeller MD, Western Institute for Neurodevelopmental Studies and Interventions, Boulder. JournalChildNeurol. [internet]. 2004 dic. [citado 24 marzo 2017]; 19(10): 798-814. Disponible en:

h t t p : / / w w w m ed s cape.com / viewarticle/495640_3

3. Argelia RM, Edgar PV, Daniel OS. JournalNeurosc. [internet] $2016 \mathrm{dec}$ [citado 24 marzo 2017]; 10: 565. Disponible en:

https://www.ncbi.nlm.nih.gov/pmc/articles/ PMC3016271/

4. Rubén O. Scandar, fundación TDAH, Jornada de actualización en TDAH, Hospital Ramón y Cajal, Madrid, España [Internet], 2009. [citado 3 oct 2016]. Disponible en: http://www.fnc.org.ar/pdfs/scandar.pdf 
5. Deault LC. A systematic review of parenting in relation to the development of comorbidities and functional impairments in children with attention-deficit/hyperactivity disorder (ADHD). Child psychiatry and human development [internet] 2010 oct [citado 20 abril 2017];41(2): 168-92.

6. Burghi G, Berrutti D, Manzanares W. Prevalencia del déficit de atención e hiperactividad. Rev. Neurología [internet] 2005 [citado 3 octubre 2016]; 40 (1): 11-15. Disponible en: https://www.researchgate.net/profile/Mateu_ Servera/publication/7997946_The_prevalence_of_attention_deficit_hyperactivity_disorder/links/0912f50eaf181e37f9000000.pdf

7. Guilherme P, Mauricio S, Bernardo L, Joseph B, Luis R, Rev. American Journal Of Psychiatry [internet], 2007 jun [citado 4 octubre 2016]; 164(6): 942-948. Disponible en:

http://ajp.psychiatryonline.org/doi/ ref/10.1176/ajp.2007.164.6.942

8. Manuela García Quiroga, Margarita Ibáñez Fanes. Terapia psicológica. RevScielo [internet] 2007 abril [citado 20 abril 2017];25(2): 123134. Disponible en: http://www.scielo.cl/scielo.php?pid=S0718-48082007000200003\&script=sci_arttext

9. Flora de la Barra. Epidemiología de trastornos psiquiátricos en niños y adolescentes: Estudios de prevalencia, Rev. Chil. Neuro-psiquiatría [internet], 2009 [citado 11 nov 2016]; 47(4): 303-314. Disponible en:

http://www.scielo.cl/scielo.php?pi$\mathrm{d}=$ S071792272009000400007\&script=sci_ arttext\&tlng=pt

10. Alfonso UM, Marcos DS, Andrea CC, Mireya RB, Jael QE. Trastorno por Déficit de Atención con Hiperactividad en Niños Escolarizados, Rev. Chil Pediatría [internet] 2009 [citado 11 nov 2016;, 80(4): 332-338. Disponible en: http://www.scielo.cl/scielo.php?script=sci_arttext\&pid=S03704106200900040004

11. Lindblad HA. ADHD after fetal exposure to maternal smoking. JournalNicotine Res [internet] 2010 [citado 20 abril 2017]; 12: 408-15. Disponible en: http://roderic.uv.es/ bitstream/handle/10550/43025/Tesis\%20 TDAH\%20CRJ.pdf?sequence $=1$

12. Baumgarnerd D., Shereiber A., Geographic Analysis of Diagnosis of Attention-Deficit/ Hyperactivity Disorder in Children: Eastern Wisconsin, USA. Rev. internacional de Psiquiatria [internet] 2014 dic [citado 4 oct 2016]: 40(4): 363-382. Disponible en: http:// ijp.sagepub.com/content/40/4/363.short

13. Harris MN, Voigt RG, Barbaresi WJ, Voge GA, Killian JM, Weaver AL, et al. ADHD and learning disabilities in former late preterm infants: a population-based birth cohort. Pediatrics [internet] 2013 [citado 21 abril 2017]; 132(3): 630-6. Disponible en: https://www. ncbi.nlm.nih.gov/pubmed?cmd=Link \&dbFrom=PubMed\&from_uid=21859915

14. Russell G, Rodgers LR, Ukoumunne, O.C. Prevalence of Parent-Reported ASD and ADHD in the UK: Findings from the Millennium Cohort Study. J Autism Dev Disord. 2014; 44(1): 31-40.

15. Wolraich M, Visser S, Bard D, Cuffe S, Neas B, Geryk L, Doffing M, Bottai M, Abramowitz A, Beck L, Holbrook J, Danielson M, The Prevalence of ADHD: Its Diagnosis and Treatment in Four School Districts Across Two States, J Autism Dev Disord. 2014; 44(1): 50-58.

16. Silva D, Colvin L, Hagemann E, Bower C. Environmentalrisk factors by gender associated with attention-deficit/hyperactivity disorder. Pediatrics [internet] 2014 ener [citado 21 abril 
2017]; 133(1): 14-22. Disponible en: https:// www.ncbi.nlm.nih.gov/pubmed/24298003

17. Abreu JA, Collado JE, Liriano HM. Trastorno por Déficit de Atención e Hiperactividad en Centros Educativos del Distrito 08-04 del Municipio Santiago. Investigación Realizada según la Escala de Brown en Estudiantes de Octavo Grado en enero-abril 2008. República Dominicana: Pontificia. Universidad Católica Madre y Maestra de Santiago; 2008.

18. Comorbilidad entre Trastorno por déficit de atención con hiperactividad y pandas en niños de 6 a 12 años en la escuela Herminia Pérez viuda de Pimentel, en Santiago de los Caballeros, enero-mayo 2011 Rev Anales de Medicina de PUCMM [Internet] 2011 oct [citado 4 de oct 2016]; 2(1): 79-88. Disponible en: http:// www.pucmm.edu.do/publicaciones/Documents/revista-anales-medicina/AMP\%20 V4N1.pdf\#page $=5$

19. Italian Journal of Pediatrics [Internet] 2010 [citado 24 marzo 2017]; 36: 79. Disponible en:

http://ijponline.biomedcentral.com/ articles/10.1186/1824-7288-36-79

20. Van der Meer D, Hartman CA, Van Rooij D, Franke B, Heslenfeld DJ, Oosterlaan J, Faraone SV, Buitelaar JK, Hoekstra PJ. RevJournal of psychiatryneuroscence [internet] 2017 [citado 20 abril 2017]; 42(2): 113-121. Disponible en: http://www.postersessiononline.com/ doi/10_3252-TDAH_es2008115.pdf 